\title{
A patient with fever and an abnormal roentgenogram
}

\author{
R.I. Cohen*, L.J. Rossoff
}

\section{Case history}

A 38 year old white woman was admitted to hospital with a one week history of right leg cellulitis, that had progressed despite treatment with dicloxacillin, $500 \mathrm{mg}$ orally, 4 times daily. She had been diagnosed as having acquired immune deficiency syndrome (AIDS) 4 years prior to admission, with sexual contact being the risk factor as there was no history of intravenous drug abuse or transfusions. Two years prior to admission, she developed immune thrombocytopenic purpura requiring splenectomy and the continued use of oral steroids. Four months prior to admission, she was diagnosed as having non-Hodgkins lymphoma and received two courses of chemotherapy with good response. Her medication at the time of admission included: dicloxacillin, fluconazole, zidovudine and prednisone $60 \mathrm{mg}$ daily, in addition to monthly inhaled pentamidine. She denied exposure to tuberculosis in the past and there was no history of recent travel.

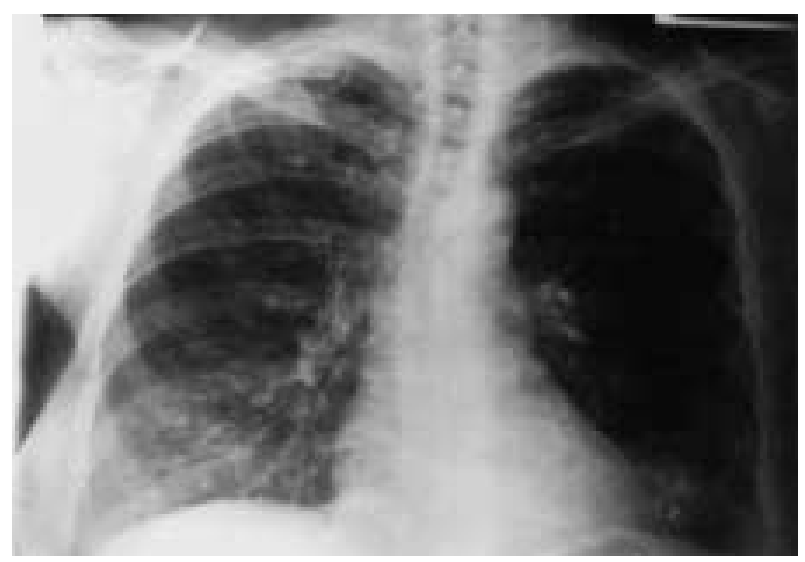

Fig. 1. - Posteroanterior (PA) chest roentogram.

Correspondence: *R. Cohen, Division of Pulmonary and Critical Care Medicine, Room C20, Long Island Jewish Medical Center, New Hyde Park, New York, 11042, USA.
The patient appeared acutely ill. Blood pressure was $100 / 60 \mathrm{mmHg}$; pulse rate was 110 beats $\cdot \mathrm{min}^{-1}$; and oral temperature $40.5^{\circ} \mathrm{C}$. Examination revealed a $3 \times 4 \mathrm{~cm}$ abscess of the right calf and surrounding cellulitis. The $\mathrm{Hb}$ was $128 \mathrm{~g} \cdot \mathrm{l}^{-1}$ with a white blood cell (WBC) count of $7.3 \times 10^{9} \cdot l^{-1}$; with $71 \%$ neutrophils, $8 \%$ band cells and $21 \%$ lymphocytes. The CD4 count was $0.184 \times 10^{9} \cdot l^{-1}$. The platelet count was $11 \times 10^{9} \cdot l^{-1}$. The lactate dehydrogenase (LDH) was normal. Blood, urine and sputum cultures were negative as was the Cryptococcal serology. The patient was anergic.

Chest X-ray and computed tomographic (CT) scan are shown below. Note that the findings did not change with the CT scan repeated in the prone position.

BEFORE TURNING THE PAGE: INTERPRET THE ROENTGENOGRAM AND THE CT SCAN AND SUGGEST THE DIAGNOSIS AND TREATMENT.

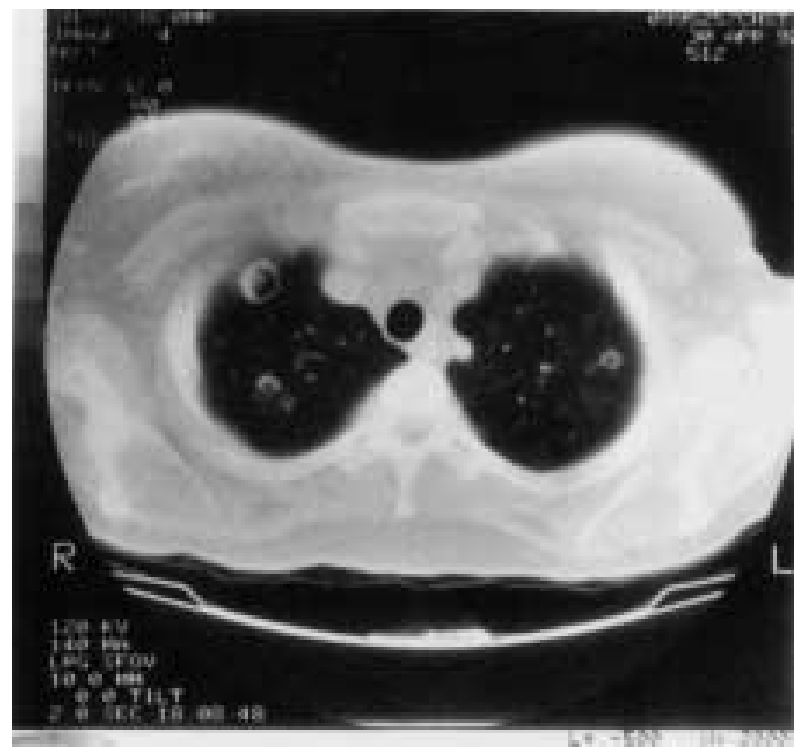

Fig. 2. - Computed tomographic (CT) scan of the chest in supine position. 


\section{Interpretation of roentgenogram and CT scan}

The chest X-ray shows an ill-defined right apical cavity. The CT scan shows bilateral, multiple, moderately thick cavities with "Target Lesions".

\section{Diagnosis}

A presumptive diagnosis of septic emboli was made, based on CT and clinical findings. This was supported by the drainage of $30 \mathrm{cc}$ of pus from the right leg abscess. Gram staining revealed Gram-positive cocci, subsequently identified as Staphylococcus aureus.

\section{Treatment}

Intravenous vancomycin, $1 \mathrm{~g}$ every $12 \mathrm{~h}$.

\section{Discussion}

The appearance of multiple cavities in this asplenic patient with AIDS, lymphoma, on high dose steroids and recent chemotherapy presented a difficult diagnostic problem. Her thrombocytopenia mitigated against invasive diagnostic procedures, such as bronchoscopy or transthoracic biopsy. Open lung biopsy was prevented by failure to significantly raise the platelet count, despite high dose steroids and multiple platelet transfusions. Thus, management was directed on clinical and radiological findings.

A presumption of septic emboli was supported by the identification of a "Target Sign", previously described by ZeLEFSKY and LutZKER [1]. This sign consists of a gravity independent density within a pulmonary cavity. We believe that we have demonstrated the same phenomenon on CT scan. The target appearance of the septic emboli is hypothesized to be a development of centrifugal spread of oedema and inflammation into the surrounding interstitium, causing a check-valve bronchiolar obstruction. The infected nidus, fixed at the site of embolization, appears suspended because it is surrounded by hyperinflated lobules [1, 2].
The Target Sign must be distinguished from that of the "air crescent". The latter may be found with mycetomas, cavitary tuberculosis and even invasive aspergillosis; all diagnostic considerations in this case $[2,3]$. The contents of the air crescent cavity appear gravity-dependent on the lateral decubitus or prone radiograph and CT scan $[1,2]$. Non-Hodgkin's lymphoma of the lung may also cavitate without the characteristics of the Target Sign, and is usually associated with extensive mediastinal and bronchopulmonary adenopathy, not seen here [4]. Cavitary upper lobe lesions have been reported with Pneumocystis carinii in patients receiving aerosolized pentamidine [5]. This and other infectious aetiologies were thought unlikely in view of the rapid clinical response to vancomycin alone.

Thus, the finding in this patient of a staphylococcal leg abscess and new onset multiple lung cavities with a Target Sign directed us to the diagnosis and treatment of septic pulmonary emboli. It allowed us to narrow the focus of antibiotic treatment and avoid invasive procedures in this immunocompromised patient.

The patient improved clinically. A follow-up CT of the chest performed 6 weeks later showed resolution of the lung cavities.

Keywords: Pulmonary cavitation, pulmonary emboli, target sign

\section{References}

1. Zelefsky MN, Lutzker LG. The Target Sign: new radiologic sign of pulmonary emboli. Am J Roentgenol 1977; 129: 453-455.

2. Silingardi V, Canossi GC, Torelli G, Russo GL. The radiologic "Target Sign" of septic pulmonary embolism in a case of acute myelogenous leukemia. Respiration 1988; 42: 61-66.

3. Gefter WB, Albelda SM, Talbot GH, Gerson SL, Cassileth PA, Miller WT. Invasive pulmonary aspergillosis and acute leukemia: limitation in the diagnostic utility of the air crescent sign. Radiology 1985; 157; 605610.

4. Bragg DG. Radiology of the lymphomas. Curr Probl Diagn Radiol 1987; 16: 183-206.

5. Murrey JF, Mills J. Pulmonary infectious complications of human immunodeficiency virus infection. Am Rev Respir 1990; 141: 1356-1372. 